\title{
Atividade física de lazer e estágios de mudança de comportamento em professores universitários
}

\author{
Edio Luiz Petroski \\ Marcelle M. de Oliveira
}

htpps://doi.org/10.5628/rpcd.08.02.209
Núcleo de Pesquisa em Cineantropometria

e Desempenho Humano (NuCIDH)

Universidade Federal de Santa Catarina (UFSC)

Brasil

\section{RESUMO}

Com objetivo de avaliar os hábitos de atividade física de lazer (AFLZ) e os estágios de mudança de comportamento relacionados à prática de atividade física, em professores universitários, foi feito um estudo descritivo de corte transversal, realizado na Universidade Federal de Santa Catarina, em Florianópolis (SC). Participaram 190 professores com dedicação exclusiva (113 do sexo masculino e 77 do sexo feminino), e foram utilizados dois questionários auto-aplicáveis: o de Godin Leisure-Time Exercise Questionnaire para o nível de atividade física de lazer, e o de Marcus para EMC. Observou-se que $71 \%$ da amostra foram classificadas como pouco ativa ou inativa fisicamente. Os EMC mais prevalecentes foram: o de Manutenção (61 casos $=32 \%$ ) e o de Contemplação (59 casos $=31 \%$ ). Foi observada associação positiva entre AFLZ e EMC e entre as idades (menores que 50 anos) e a AFLZ ( $p<0,05)$. Como conclusões: o nível de AFLZ de professores universitários com dedicação exclusiva tende ser reduzido e irregular; os a prática de AFLZ sugere que homens realizam mais atividades físicas vigorosas e leves e as mulheres mais as moderadas.

Palavras-chave: professores universitários, atividade física, comportamento, sedentarismo, lazer.

\author{
ABSTRACT \\ Leisure-time physical activity and \\ behavior change stages in University teachers
}

The objective was to evaluate leisure-time physical activity (LTPA) and behavior change stages (BCS) related to physical activity among University teachers. A descriptive, cross-sectional study was carried out at the Universidade Federal de Santa Catarina, in Florianópolis, SC, Brazil enrolling 190 fulltime teachers (113 males and 77 females).

Two self-administered questionnaires were used, the Godin Leisure-Time Exercise Questionnaire was used to assess LTPA levels; and the Marcus questionnaire was used for BCS. It was observed that $71 \%$ of the sample was classified as either performing little activity or being regularly inactive. The two most prevalent BCS were: the Maintenance stage, with 61 cases (32\%), and the Contemplation stage, with 59 cases $(31 \%)$, in which men were more prevalent. The results demonstrated a positive association between LTPA and BCS and between ages below 50 years and LTPA $(p<0.05)$. Conclusions -1 . Fulltime University teachers' level of LTPA was low and irregular; 2. Analysis of LTPA practices indicated that the men performed more vigorous and light activities while the women performed more moderate activities.

Key-words: university teachers, physical activity, behavior, inactivity, leisure. 


\section{INTRODUÇÃo}

Estima-se que a inatividade física é um fator de risco independente para doenças crônicas, e que contribui com aproximadamente 1,9 milhões de mortes por ano em todo o mundo(42). O sedentarismo é epidêmico em sociedades industrializadas porque o comportamento do ser humano tem propiciado e perpetuado este estilo de vida há dois séculos, e assim, pode-se entender a inatividade física como um problema social, mais do que uma questão de escolha individual.

Para Yancey et al.(43) a função essencial da atividade física tanto como um independente fator de proteção contra inúmeras doenças crônicas (como diabetes tipo $2(6,38,41)$, cardiopatias $(6,13)$, certos tipos de câncer $^{(21,22)}$, osteoporose ${ }^{(19)}$ e depressão(16), tornando-se um saudável fator de controle ponderal está ganhando reconhecimento científico crescente, e muito embora os estudos sobre promoção da atividade física tenham avançado velozmente, a prática da promoção da $\mathrm{AF}$ em meio à população ainda dá os primeiros passos. Pesquisas sobre a inatividade física em diferentes subgrupos da população brasileira indicam prevalências entre $40 \%$ a $87 \%(5,15,17,27,29,31)$.

Recentemente, a atividade física no tempo de lazer (AFLZ) tem recebido expressiva atenção devido ao fato de existir menor atividade física no trabalho nos países industrializados (pela tecnologia e automação presentes nas tarefas laborais), o que a torna uma medida que representa mais fielmente a AF praticada pela população economicamente ativa ${ }^{(8)}$.

Caspersen et al.(9) citam que sujeitos que relatam não praticar AFLZ possuem níveis menores de $\mathrm{AF}$ na vida diária, sugerindo que a AFLZ é a mais forte e incontestável "dimensão" de prática de AF relacionada à inatividade física (quanto menor o nível de AFLZ, maior o nível de sedentarismo).

Em 1996, o Colégio Americano de Medicina do Esporte e o Centro de Prevenção e Controle de Doenças ${ }^{(30)}$ desenvolveram guias para quantificar o montante de atividade física, requerida para propiciar benefícios à saúde, preconizando a execução de AF moderadas em pelo menos 30 minutos diários, todos dias da semana, de preferência, ou pelo menos em três dias. As sessões de AF não precisam ser de esforços continuados, podem ser realizados em pequenas sessões (três vezes de 10 minutos, por exemplo, ou duas vezes de 15 minutos).

É nesse contexto que se desenvolve e aprimora estudos relacionados ao comportamento humano e à $\mathrm{AF}$, considerando-se a necessidade de desenvolver estratégias de intervenção e promoção da prática de AF regulares, com vistas à adoção e manutenção de comportamentos saudáveis $(34,40)$.

Para que se desenvolva uma intervenção efetiva em AF, é importante o conhecimento dos fatores que possibilitam as mudanças de comportamento de uma população. O Modelo Transteorético de Mudança de Comportamento(32) tem sido freqüentemente utilizado para tal finalidade. Conhecido também como "Modelo de Estágios de Mudança", ele descreve como as pessoas movem-se através de uma série de cinco estágios distintos, à medida que avaliam a informação e a relevância da atividade física para suas vidas. Os estágios descritos nesse modelo são o de Pré-contemplação (o indivíduo não pretende modificar seu comportamento num futuro próximo), Contemplação (existe a intenção de mudar, mas não logo), Preparação (existe a intenção de mudar nos próximos seis meses), Ação (mudanças recentes no comportamento) e Manutenção (as mudanças no comportamento já são mantidas por mais de seis meses).

Contudo, mudanças profundas de comportamento (tais como modificações do estilo de vida) exigem antes de tudo compreensão da situação externa (o que ocorre onde o sujeito está inserido), e depois um movimento interno - e, normalmente, lento em direção às modificações de hábitos.

Este estudo teve como objetivo verificar os hábitos de AFLZ e os estágios de mudança de comportamento relacionados à prática de atividade física, em indivíduos com nível educacional superior, cujos trabalhos supostamente requerem demanda energética leve. Assim, pretende-se estudar o padrão de comportamento de indivíduos com grau de instrução superior, pois este fator (instrução, escolaridade e acesso a informações sobre exercício) pode influenciar o comportamento relacionado à atividade físi$\mathrm{ca}^{(11)}$, assim como pretende-se investigar adultos cujas atividades laborais não requeiram esforços físicos, uma vez que trabalhos de natureza pouco ativa podem estar relacionados à inatividade física, tanto por implicar baixo consumo energético (atividade 
pouco acima do gasto energético de repouso) como por propiciar uma atitude pouco ativa em grande parte do dia(35).

\section{METODOLOGIA}

Este estudo caracteriza-se como descritivo, de corte transversal, realizado na Universidade Federal de Santa Catarina (UFSC), em Florianópolis, SC.

A seleção da amostra deu-se por amostragem sistemática (aleatória), e ocorreu da seguinte maneira: 1. obteve-se uma lista de nomes dos professores junto ao Departamento de Recursos Humanos; 2. extraiu-se da referida lista, os professores afastados, pensionistas e inativos, obtendo-se a listagem de nomes dos professores com dedicação exclusiva $(n=1354)$ que constituiria a população de estudo; 3 . calculou-se o tamanho mínimo da amostra admitindo-se erro amostral máximo de $5 \%$, utilizando-se a fórmula de Barbetta ${ }^{(4)}$, obteve-se como tamanho da amostra $\mathrm{n}=309$.

Após a distribuição dos questionários em todos os departamentos da UFSC e a todos os nomes selecionados, retornaram para análise 190 questionários, elevando o erro amostral para 7\%. Finalmente, participaram do estudo 190 professores, sendo 113 do sexo masculino e 77 do sexo feminino.

\section{Coleta de dados e Instrumentos}

Utilizou-se um questionário auto-aplicável, composto por quatro partes distintas: questões demográficas (idade, sexo, estado civil e titulação acadêmica); nível sócio-econômico; prática de atividade física habitual no tempo de lazer; estágio de mudança de comportamento frente à atividade física. No questionário, foram incluídos uma carta de apresentação da pesquisa e o termo de consentimento livre e esclarecido. A classificação sócio-econômica seguiu as orientações da Associação Brasileira de Empresas de Pesquisa (ABEP) (2). O critério de Classificação Econômica Brasil, estabelece sete classes econômicas (A1, A2, B1, $B 2, C, D, E)$, que definem do maior ao menor poder de compra das pessoas e das famílias urbanas. Os dados quanto à Prática de Atividade Física Habitual no Tempo de Lazer foram obtidos através do Godin Leisure-Time Exercise Questionnaire(14), no qual o sujeito deveria informar o número de vezes em que praticou atividades físicas de intensidade vigorosa (AFV), moderada (AFM) e leve (AFLZ), conside- rando um período de sete dias habituais, durante pelo menos 15 minutos diária. O número de vezes declarado para cada tipo de atividade foi então multiplicado por determinado coeficiente de esforço, equivalente ao gasto energético da referida atividade ("9" para AFV; "5" para AFM; "3" para AFL), conforme equação a seguir: Atividade Física de Lazer semanal $=(9 \mathrm{xAFV})+(5 \times \mathrm{AFM})+(3 \mathrm{x} \mathrm{AFL})$. Segundo Godin e Shephard(14), a objetividade deste instrumento é de 0,94 para atividades vigorosas e $0,74(\mathrm{p}<0,05)$ para total de AF praticada $(\mathrm{AFL}+$ $\mathrm{AFM}+\mathrm{AFV})$.

Desta forma, admitiu-se que o indivíduo que obteve ao menos 15 pontos no instrumento Godin LeisureTime Exercise Questionnaire e respondeu "sim" à questão complementar, possui um padrão de prática de AF regular, uma vez que os 15 pontos refletem, no mínimo: a) cinco declarações de prática de AFL coeficiente $3 \times 5$ declarações $=15$ pontos; ou b) três declarações de prática de AFM - coeficiente $5 \times 3$ declarações $=15$ pontos; ou c) duas declarações de prática de AFM e duas declarações de prática de AFL - (coeficiente $5 \times 2$ declarações) + (coeficiente $3 \times 2$ declarações) $=16$ pontos.

Para a avaliação geral dos sujeitos, considerou-se a pontuação total obtida (em escala ordinal) e a pontuação dividida em categorias de quinze em quinze pontos $(00-14 ; 15-29,30-44 ; 45-59$; 60 pontos ou mais), utilizada para medir a correlação desta variável com as demais variáveis categóricas do estudo. Assim, foi possível, ainda, gerar um escore para classificar o padrão de AFLZ, considerando os sujeitos como inativos ou insuficientemente ativos (atividade física semanal < que 30 pontos) e ativos regularmente (atividade semanal $\geq 30$ pontos).

A última parte do questionário utilizado destinavase às informações sobre os Estágios de Mudança de Comportamento (EMC), na qual foi utilizada a escala de respostas categóricas desenvolvida por Marcus(23).

Os questionários foram coletados (retorno) na secretaria do departamento do respectivo professor, no quinto e no décimo dia subsequente à data de sua distribuição em cada Centro de Ensino da UFSC. Os aspectos éticos foram respeitados e todos os participantes assinaram o termo de consentimento livre e esclarecido. 
Os dados foram registrados em um banco de dados, utilizando-se o programa Excel ${ }^{\circledR} 97$ (ambiente Windows ${ }^{\circledR}$ ) e o programa Statistica ${ }^{\circledR}$ (versão 5 ).

Efetuou-se o tratamento dos dados através da distribuição de freqüências simples e conjunta, percentuais, cálculo de médias, medianas, desvio padrão e valores máximos e mínimos. Foram ainda realizados os testes de associação (Qui-quadrado) entre as variáveis categóricas e a correlação (Spearman) entre as variáveis ordinais.

\section{RESULTADOS}

A Tabela 1 apresenta as principais características demográficas e sócio-econômicas dos sujeitos investigados. A amostra caracteriza-se como sendo constituída prioritariamente por indivíduos do sexo masculino $(59,5 \%)$, embora a quantidade de mulheres tenha sido expressiva $(40,5 \%)$. O estado civil predominante é o casado (65,8\% dos casos). Quanto à idade, a amostra constitui-se de adultos jovens e de meia-idade, sendo a média de $44,8 \pm 6,8$ anos (mínimo 27,9 anos e máximo 66,6 anos). A maior parte dos indivíduos situa-se entre 40 e 48 anos, e as duas faixas etárias de maior concentração de casos são as faixas de 35 a 44 anos e 45 a 54 anos. A formação acadêmica mais prevalecente é a de doutorado. Especificamente quanto à titulação dos respondentes, verifica-se que $93 \%$ deles têm pós-graduação ( $5,1 \%$ são especialistas, $30,5 \%$ são mestres e 57,4 são doutores). Dos 190 indivíduos, 10 (5,2\%) cursaram somente a graduação e quatro $(2 \%)$ não informaram.

A maioria dos indivíduos $(65,8 \%)$ situou-se nas classes B1 e A2, segundo a ABEP (2) caracterizando-se como amostra de bom nível sócio-econômico.

A Tabela 2 mostra a classificação do nível de AFLZ, na qual se observa que $71 \%$ da amostra foi classificada como pouco ativa ou inativa regulamente, e 29\% como ativa regularmente.

Foram observadas 134 citações para prática de AF leves (71\%), 129 citações para prática de AF moderadas (68\%), e 55 citações para prática de AF vigorosas (29\%). Como a prática de AF de determinada intensidade não exclui a possibilidade de praticar as demais, observou-se ainda 32 citações para prática dos três tipos de AF (vigorosas, moderadas e leves), sendo que 41 sujeitos praticavam AF vigoro-
Tabela 1. Características demográficas e socioeconômicas de professores universitários da UFSC.

\begin{tabular}{|c|c|c|}
\hline Características & $n$ & $\%$ \\
\hline \multicolumn{3}{|l|}{ Sexo } \\
\hline Masculino & 113 & 59,5 \\
\hline Feminino & 77 & 40,5 \\
\hline \multicolumn{3}{|l|}{ Faixa etária } \\
\hline $25-34$ & 14 & 7,3 \\
\hline $35-44$ & 68 & 35,8 \\
\hline $45-54$ & 83 & 43,7 \\
\hline $55-65$ & 09 & 4,7 \\
\hline 66 ou mais & 01 & 0,6 \\
\hline \multicolumn{3}{|l|}{ Estado Civil } \\
\hline Casado & 125 & 65,8 \\
\hline Solteiro & 44 & 23,2 \\
\hline Divorciado & 16 & 8,4 \\
\hline Viúvo & 3 & 1,6 \\
\hline Não informou & 2 & 1 \\
\hline \multicolumn{3}{|l|}{ Titulação } \\
\hline Doutorado & 108 & 57,4 \\
\hline Mestrado & 58 & 30,5 \\
\hline Especialização & 10 & 5,1 \\
\hline Graduação & 10 & 5,1 \\
\hline Não informou & 4 & 2 \\
\hline \multicolumn{3}{|l|}{ Nível Socioeconômico* } \\
\hline A1 (classe econômica alta) & 20 & 10,5 \\
\hline A2 (classe econômica alta) & 72 & 37,9 \\
\hline B1 (classe econômica média) & 53 & 27,9 \\
\hline B2 (classe econômica média) & 39 & 20,5 \\
\hline C (classe econômica baixa) & 06 & 3,2 \\
\hline
\end{tabular}

*ABEP(2) - estabelece sete classes socioeconômicas (A1, A2, B1, B2, C, D, E) do maior ao menor poder de compra das pessoas e das famílias urbanas.

Tabela 2. Nível habitual atividade física de lazer de professores universitários.

\begin{tabular}{lccc}
\hline & Categoria (pontos) & $\mathrm{n}$ & $\%$ \\
\hline Pouco ativos ou inativos & Até 14 pontos & 70 & 36,8 \\
\cline { 2 - 4 } & $15-29$ & 65 & 34,2 \\
\hline Ativos regularmente & $30-44$ & 37 & 19,5 \\
\cline { 2 - 4 } & $45-59$ & 08 & 4,2 \\
\cline { 2 - 4 } & 60 ou mais & 10 & 5,3 \\
\hline Total & & 190 & 100 \\
\hline
\end{tabular}


sas e leves, 44 praticavam AF vigorosas e moderadas, e 93 praticavam AF moderadas e leves. Quando avaliadas as associações entre as variáveis idade (faixa etária; menor que 50 anos; idade decimal), gênero e AFLZ, observou-se que há associação somente entre idades menores que 50 anos e a $\operatorname{AFLZ}\left(\chi^{2}=9,62, \mathrm{P}=0,04\right)$ e gênero (somente mulheres) e AFLZ $\left(\chi^{2}=7,88 ; \mathrm{P}=0,05\right)$. Apesar de não serem muito expressivas, as associações são estatísticamente significativas e não ocorreram ao acaso ( $\mathrm{p}<0,05)$.

Os resultados por faixa etária indicam que em todas predomina o número de pessoas que não participa de qualquer programa de AF, embora 78 (41\%) sujeitos tenham afirmado praticar AFLZ, a maior parte deles da faixa etária de 45-54 anos. A maior proporção de sujeitos irregularmente ativos encontra-se na faixa etária de 35-44 anos. A única faixa etária em que houve predomínio de indivíduos regularmente ativos foi a de 45-54 anos.

As práticas mais citadas foram: caminhadas e natação (ambos com 21 citações); hidroginástica (13 citações) e musculação (12 citações). As demais práticas relacionadas incluem esportes coletivos, AF orientadas e não-orientadas (andar de bicicleta, alongamento, correr, surfar, passeios ecológicos) e dança. Quando analisados os dados de Estágios de Mudança de Comportamento (EMC), mostram-se resultados similares aos de AFLZ. Com efeito, tanto com o teste Qui-quadrado como com o de correlação de Spearman, verificou-se associação positiva estatísticamente significativa entre as variáveis EMC e AFLZ ( $p=0,000$ em ambos os casos) sugerindo que maiores níveis de AFLZ estão moderadamente relacionados a estágios de comportamento de maior atividade física.

Foram seis os Estágios de Mudança de Comportamento considerados neste estudo. A Figura 1 ilustra a distribuição de freqüências absolutas para cada estágio, e permite identificar os dois estágios com maior ocorrência de casos: o estágio de Manutenção, com 61 casos (32\%), e o estágio de Contemplação, com 59 casos (31\%).

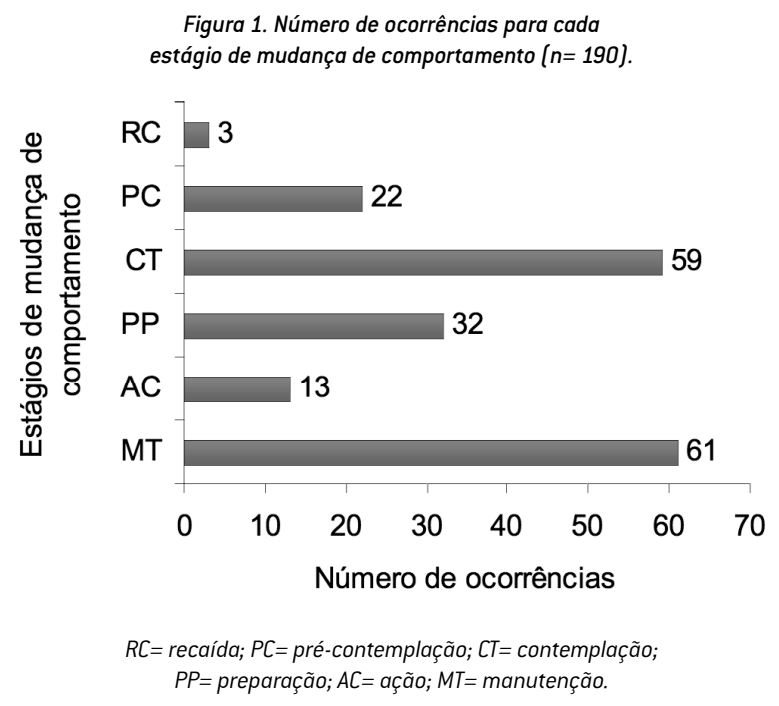

Na Tabela 3, é possível observar a distribuição conjunta de freqüências segundo o EMC, considerandose também o padrão de prática de AF habitual, embora não se tenha detectado associação ( $\mathrm{p}>$ $0,05)$ entre as variáveis.

Tabela 3. Estágios de mudança de comportamento (EMC) e padrão de AFLZ de professores universitários.

\begin{tabular}{lccccc}
\hline \multirow{2}{*}{ EMC } & \multicolumn{2}{c}{ Mulheres } & \multicolumn{2}{c}{ Homens } \\
\cline { 2 - 6 } & & $\mathbf{n}$ & $\%$ & $\mathrm{n}$ & $\%$ \\
\hline \multirow{4}{*}{ EMC } & Recaída - RC & 1 & 1,3 & 2 & 1,8 \\
\cline { 2 - 6 } & Pré-contemplação - PC & 6 & 7,7 & 16 & 14,2 \\
\cline { 2 - 6 } & Contemplação - CT & 19 & 24,7 & 40 & 35,4 \\
\cline { 2 - 6 } & Preparação - PP & 16 & 20,8 & 16 & 14,2 \\
\cline { 2 - 6 } & Ação - AC & 6 & 7,8 & 7 & 6,2 \\
\cline { 2 - 6 } Padrão & Manutenção - MT & 29 & 37,7 & 32 & 28,3 \\
\cline { 2 - 6 } de AFLZ & Pouco ativo ou inativo & 42 & 54,5 & 74 & 65,5 \\
\cline { 2 - 6 } & Ativos regularmente & 35 & 45,5 & 39 & 34,5 \\
\hline
\end{tabular}

É importante ressaltar que a classificação do padrão de AF "pouco ativos ou inativos" corresponde a indivíduos nos estágios de Recaída, Pré-contemplação, Contemplação e Preparação (a maior parte dos casos em ambos os gêneros), enquanto que os "ativos regularmente" correspondem àqueles nos estágios Ação e Manutenção. 


\section{DISCUSSÃO}

Os escores obtidos para o AFLZ através do instrumento Godin Leisure-Time Exercise Questionnaire (14) apontaram reduzida prática de AF, pois verificou-se que $71 \%$ dos professores da UFSC eram inativos ou insuficientemente ativos, sendo as AF Leves (AFL) as mais praticadas, e mesmo assim somente 2,4 vezes por semana, em média. As AF Moderadas (AFM) são praticadas duas vezes na semana, em média, e as AF Vigorosas (AFV) menos de uma vez por semana, e somente 32 indivíduos (16,9\%) relataram praticar as três intensidades de AF durante uma semana habitual. O número de vezes de prática semanal apresenta-se baixo, o que indica padrões de AF insatisfatórios, abaixo do mínimo proposto na literatura.

Segundo as recomendações atuais para prática de $\mathrm{AF}$ do American College of Sports Medicine ${ }^{(30,41)}$, o montante de prática de AF semanal deve ser em torno de cinco vezes ou mais por semana, durante períodos de 30 minutos contínuos ou intermitentes para AFM, ou três vezes por semana, durante 20 minutos contínuos ou mais para AFV.

De maneira geral, os resultados referentes à prática de AF apontam para um comportamento pouco voltado à saúde, e ainda menos à aquisição ou manutenção de aptidão física, porque as AFL e AFM são as mais praticadas (com ênfase para as AFL). A maior parte dos que praticam alguma AF, fá-lo sumariamente, sem atingir o mínimo necessário para trazer benefícios à saúde.

Os resultados sobre as atividades físicas de lazer obtidos nesta pesquisa, confirmam dados de outros estudos quanto às diferenças de padrão de prática de homens e mulheres, pois, como relatado na literatura, AF mais intensas (vigorosas) são praticadas principalmente por homens, já as mulheres realizam mais AFM(28). Também observou-se que $18,6 \%$ dos homens pesquisados praticam os três tipos de $\mathrm{AF}$ (vigorosa, moderada e leve), contra somente 10,5\% das mulheres. Existem evidências de que parece haver grande variabilidade interna e externa entre países desenvolvidos e em desenvolvimento(12), e que as mulheres são insuficientemente ativas, aparentemente em qualquer idade, mas que isso pode ser devido mais a fatores sócio-culturais que propriamente a fatores biológicos, dados não confirma- dos no presente estudo. Diversos estudos relatam que mulheres praticam menos AF no lazer que os homens ${ }^{(10,34)}$ e há evidências de que, com o avanço da idade cronológica, aumentam as probabilidades das mulheres serem insuficientemente ativas (26). Por exemplo, com relação aos fatores sócio-ambientais e demográficos de estilo de vida, Jurj et al.(18) verificaram que as mulheres estão duas vezes mais propensas a ser fumantes, consumir regularmente álcool, chá e "ginseng" e exercitar-se regularmente se os maridos têm os mesmos hábitos.

Considerando-se este estudo, pode-se afirmar que existiram diferenças importantes entre homens e mulheres quanto aos padrões de AF: a) os homens incluem em sua rotina, mais que as mulheres, AF de intensidades diversas; $b$ ) os homens praticam mais AFV que as mulheres; c) as mulheres detêm-se mais na prática de AFM e AFL; d) a prática de AF das mulheres está mais relacionada à participação em AF de lazer programadas e sistematizadas que a dos homens (grupos de exercício, academias, caminhadas, entre outros).

Todavia, focalizando a distribuição de gênero em cada estágio de comportamento, nota-se que as mulheres concentram-se no estágio de Manutenção $(37,7 \%)$, enquanto os homens concentram-se no estágio de Contemplação (35,4\%). Percebe-se, ainda, que o estágio de maior similaridade entre os gêneros é o estágio de Ação. Assim, de acordo com os resultados relativos aos EMC, sugere-se maior quantidade de prática regular por parte das mulheres: é possível observar que o número de mulheres que já aderiram à prática regular de $\mathrm{AF}(37,7 \%$ das mulheres em estágio de Manutenção) é superior ao de homens $(28,3 \%)$.

Sugere-se na literatura Marcus et al.(25) que, como o exercício físico é um comportamento necessariamente voluntário e que consome tempo, ele pode "competir" com as responsabilidades vocacionais ou com outros interesses de lazer. Assim, programas estruturados podem criar barreiras adicionais para algumas pessoas, incluindo agendamento de horários, ter que se deslocar até as instalações da aula ou taxas de matrícula.

Para transformar o crescente valor que a AF tem recebido em prática (atitude ativa, comportamento fisicamente ativo), serão necessárias intervenções 
com abordagens sistêmicas, multiniveladas e multisetoriais, as quais subsidiarão tanto a possibilidade individual como a capacidade organizacional para promover mudanças de comportamento, criar novas normas sociais, além de promover mudanças políticas e ambientais, que por sua vez darão suporte ao gasto de maiores níveis de energia entre a população(43).

Especificamente quanto aos resultados dos EMC, visto que as próprias definições de cada estágio evidenciam sua estreita ligação com os padrões de AF habitual, admite-se que aqueles indivíduos engajados na prática de $\mathrm{AF}$ estariam nos estágios de ação e manutenção, com a diferença de que a manutenção implica mais de seis meses de AF praticadas regularmente. Neste estudo, aproximadamente $40 \%$ dos sujeitos declararam-se ativos fisicamente, e encontravam-se nos estágios de ação e manutenção, sendo, respectivamente, $7 \%$ e $33 \%$ dos casos.

Em um grupo de adolescentes (14 a 19 anos), 61,6\% foram classificados como inativos ou irregularmente ativos(33) - o que condiz com os dados dos adultos da presente pesquisa - e $26,2 \%$, como sedentários (pré-contemplativos e contemplativos), muito embora $38,3 \%$ dos adolescentes encontravam-se em estágios de manutenção e ação.

Os níveis de sedentarismo são similares entre homens e mulheres no Brasil e, dentre 16 cidades investigadas (15 capitais mais Distrito Federal), uma faixa de $28 \%$ a $54 \%$ da população declarou ter estilos de vida sedentários(3).

Os estágios de pré-contemplação, contemplação e preparação denotam nenhuma prática de AF regular, embora possa haver alguma iniciação de prática no estágio de preparação. Encontraram-se nestes estágios a maior parte dos professores pesquisados: $11 \%$ foram identificados como pré-contempladores, $30 \%$ como contempladores e $17,5 \%$ como estando no estágio de preparação. Isso confirma o padrão de comportamento relacionado à pouca atividade física, e esta predominância de inatividade física também é verificada em outros países $(7,20)$. Um estudo realizado com trabalhadores americanos indicou que $69 \%$ das mulheres e $64 \%$ dos homens não atingem as recomendações descritas para a atividade física no tempo de lazer(7). Veicula-se em estudos científicos que ser inativo dobra a chance de risco para doenças coronarianas e, apesar disto, a inatividade física (sedentarismo) é comum, em mais da metade da população de países industrializados (principalmente norte-americanos) sendo menos ativa do que se recomenda $(37,41)$.

A inatividade física é reconhecida como um fator de risco independente para o desenvolvimento de doenças cardiovasculares ${ }^{(24)}$, e possibilita risco para doenças do coração similar ao tabagismo, pressão alta ou alto nível de colesterol, todavia, ela é mais prevalente que quaisquer outros fatores de risco(12). Assim, é urgente a necessidade de desenvolver efetivas intervenções com vistas à promoção, adoção e manutenção da atividade física(24), haja vista que sem intervenção, muitas pessoas permanecem sedentárias(36). Neste estudo, verificou-se que os mais jovens (menos de 50 anos) praticam mais AFLZ, confirmando dados gerais. Na pesquisa de Saúde Mundial (realizada pela WHO e apresentada em 2004, "World Health Survey results in Brasil") observa-se que os níveis de sedentarismo são de cerca de $24 \%$, sendo os jovens mais ativos que os maiores de 50 anos de idade (3).

A necessidade de encorajar o estilo de vida ativo, com o avançar da idade tem sido relatado (1), pois especialmente na meia-idade praticar atividades físicas torna-se expressivamente importante, uma vez que poderia afetar a capacidade de manter-se ativo na terceira idade, fase em que o estilo de vida ativo pode ter maior impacto em prevenir ou evitar quadros de enfermidade.

Estudos brasileiros indicam elevadas prevalências de inatividade física em diferentes segmentos populacionais. Em São Paulo e Pelotas (RS) (17), a prevalência de inatividade (menos de $150 \mathrm{~min} . / \mathrm{semana}$ ) foi de aproximadamente $40 \%$. Monteiro et al.(29), utilizando uma amostra do nordeste e sudoeste, observaram prevalência (30 min. em 5 ou mais dias por semana) de $87 \%$. Na cidade de Salvador-BA, a prevalência de AFLZ foi de 72,5\%, e Gomes et al.(15) relatam prevalência AFLZ em torno de $59 \%$ para homens e $78 \%$ para mulheres maiores que 12 anos no Rio de Janeiro. Em Santa Catarina(5), observouse prevalência de AFLZ em torno de $67 \%$ entre mulheres e $34,8 \%$ entre homens, em se tratando de trabalhadores da indústria.

Convém ressaltar que, pela AFLZ, classificou-se $71 \%$ 
de sujeitos como pouco ativos, enquanto a categorização conforme o EMC aponta para cerca de $60 \%$. Estes resultados devem-se, provavelmente, a variações na interpretação do instrumento de medida para EMC, no qual o indivíduo pode auto-denominar-se regularmente ativo, ou então à descrição das AF habituais no instrumento de Godin e Shephard(14), o qual está sujeito à memorização e percepções individuais do esforço diário em AFLZ. Todavia, os dados confirmam o comportamento da amostra de forma geral, e observou-se que a mensuração do EMC está significativamente associada à mensuração do AFLZ ( $<$ 0,05).

Os resultados do presente estudo são relevantes para a saúde pública, haja vista que as informações sobre as AFLZ, bem como sobre os estágios de mudança de comportamento de professores universitários permitem o planejamento e implantação de políticas institucionais, projetos e programas para o incremento da AF no campus, com vistas a reverter ou minimizar o quadro elevado de sedentarismo. Contudo, uma limitação do presente estudo é o fato de se investigar somente as atividades físicas no tempo de lazer, e não incluir atividades de/durante o transporte e tarefas domésticas. Ressalta-se, também, que se trata de uma população específica de professores da UFSC, o que limita a extensão dos resultados a outros níveis de ensino, bem como a professores sem dedicação exclusiva.

Seria importante estimular indivíduos no estágio de contemplação a tomarem a decisão de efetivar o início da prática, passando para o estágio de preparação e, conseqüentemente, ao de ação. Da mesma maneira, dever-se-ia trabalhar para esclarecer as possibilidades de prática de AF regulares (diárias, não-sistemáticas, em sessões contínuas ou acumuladas, não necessariamente em intensidade vigorosa), a fim de que os indivíduos no estágio de preparação tornemse regularmente ativos.

Sendo a internet uma ferramenta utilizada com freqüência por professores universitários, a mesma poderia ser utilizada como meio de informação e de intervenção. $\mathrm{O}$ uso da internet tem sido fortemente recomendado para a mudança de comportamento em relação à atividade física. As evidências do potencial da web para informar e facilitar as mudanças de comportamento em relação à $\mathrm{AF}$, têm sido recentemente relatadas na literatura( ${ }^{(39)}$, estudos sugerem que sujeitos podem modificar o comportamento em relação à atividade física, revelando um aumento no padrão de atividade física e decréscimo no tempo gasto sentado(38).

\section{CONCLUSÕES}

A partir dos resultados obtidos neste estudo, puderam ser destacadas as seguintes conclusões:

1. O nível de atividade física habitual de professores universitários com dedicação exclusiva foi reduzido e irregular;

2. Distribuindo-se os casos conforme intensidades de AFLZ, indica-se que homens praticavam mais AFV e AFL e as mulheres praticavam mais AFM; 3. AFM e AFL foram, em geral, as mais freqüentes entre o grupo estudado;

4. Os homens diversificaram mais sua prática de AF, considerando a intensidade de prática;

5. Programas sistemáticos e regulares para prática de AF são constituídos, principalmente, por caminhadas e natação, seguidas de hidroginástica e musculação;

6. As mulheres são mais propensas à adesão a programas de AF no tempo de lazer;

7. A maior parte dos professores universitários está classificada em EMC que indicam prática de $\mathrm{AF}$ reduzida e irregular;

8. As mulheres são mais ativas segundo os seguintes parâmetros: regularidade de prática e o número de ocorrências em EMC de Manutenção e Ação.

\section{CORRESPONDÊNCIA}

\section{Prof. Dr. Edio Luiz Petroski}

Universidade Federal de Santa Catarina

Centro de Desportos

Departamento de Educação Física

Campus Universitário - Trindade

Cep 88.040-900 - Florianópolis, SC. Brasil

Telefone / fax : (48) 3331-8562.

E-mail: petroski@cds.ufsc.br 


\section{REFERÊNCIAS BIBLIOGRÁFICAS}

1. Abbott RD, Curb JD, Rodriguez BL, Masaki KH, Yano K. Schatz IJ, Ross GW, Petrovitch H (2002). Age-related changes in risk factor effects on the incidence of coronary heart disease. Ann Epidemiologist 3: 73-181.

2. ABEP - Associação Brasileira de Empresas de Pesquisa (2003). Critério de Classificação Econômica Brasil. Endereço eletrônico: http://www.abep.org/codigosguias/ABEP_CCEB.pdf.

3. Banco Mundial (2005). Relatório No. 32576-BR - BRASIL: Enfrentando o Desafio das Doenças Não Transmissiveis no Brasil. 15 de novembro de 2005, Unidade de Gerenciamento do Brasil, Unidade de Gestão do Setor de Desenvolvimento Humano Região da América Latina e do Caribe.

4. Barbetta PA (1998). Estatística aplicada às Ciências Sociais. 2a Ed., Florianópolis: Editora da UFSC.

5. Barros MV, Nahas MV (2001). Comportamentos de risco, auto-avaliação do nível de saúde e percepão de estresse entre trabalhadores da indústria. Rev Saúde Pública 35(6): 554-563.

6. Bassuk SS, Manson JE (2005). Epidemiological evidence for the role of physical activity in reducing risk of type 2 diabetes and cardiovascular disease. J Appl Physiol 99(2): 1193-1204.

7. Caban-Martnez AJ, Lee DJ, Fleming WG, LeBlanc WG, Arheart KL, Chung-Bridges K, Christ Sl, McCollister KE, Pitman T (2007). Leisure-time physical activity levels of the US workforce. Prev Med J 2007 (em publicação).

8. arpersenCJ, Kriska AM (1997). Introdution to a collettion of physical activity questionnires. Med Sci Sports Exerc 29(6): S5-S9.

9. Caspersen CJ, Merrit RK, Stephens T (1995). International physical activity patterns: a methodological pespective. In: Dishman RK (ed.). Advances in exercise adherence. Champaign: Human Kinetics, 73-110.

10. Dias-da-Costa JS, Hallal PC, Well JCK, Daltoé T, Fuchs SC, Menezes AMB, Olinto MTA (2005). Epidemiolgy of leisuretime physical activity: a population-based satudy in southern Brazil. Cad Saúde Pública 21(1): 275-282.

11. Dishman RK (1995). Introduction: Consensus, problems and prospects. In: Dishman RK (ed.). Advances in exercise adherence. Champaign: Human Kinetics, 01-27.

12. Dubbert PMT. Carithers, et al. (2002). Obesity, physical inactivity, and risk for cardiovascular disease. Am J Med Sci 324(3): 116-26.

13. Forrest KY, Bunker CH, Kriska AM, Ukoli FA. Huston SL, Mardovic N (2001). Physical activity and cordivascular risk factors in a developing population. Med Sci Sports Exerc 33(9): 1598-1604.

14. Godin G, Shephard RJ (1985). A simple method to assess exercise behavior in the community. Can J Applied Sport Sciences 10: 141-146.

15. Gomes VB, Siqueira KS, Sichieri R (2001). Atividade física em uma amostra probabilística na população do Município do Rio de Janeiro. Cad Saúde Pública 17(4): 969-976.

16. Gumarães JMN, Caldas CP (2006). A influência da atividade física nos quadros depressivos de pessoas idosas: uma revisão sistemática. Rev Bras Epidemiol 9(4): 481-92.

17. Hallal PC, Matsudo SM, Matsudo vhr, Araújo TL, Andrade DR, Bertoldi AD (2005). Physical activity in adults from two brazilin areas: similarities and differences. Cad Saúde Pública 21(2): 573-580.

18. Jurj AL, Wen W. Li HL, Zheng W, Yang G, Xiang YB, Gao YT, Shu XO (2005). Spousal correlations for lifestyle factors and selected diseases in chinese couples. Ann Epidemiologist (AEP) 60(7): $1-7$.
19. Kohort WM, Bloomfield AS, Little KD, Nelson ME, Yingling VR (2004). American College of Sports Medicine. American College so Sports Medicine Position Stand: Physical activity and bone health. Med Sci Sports Exerc 36(11): 1986-1996.

20. Ku P-W, Fox KR, MacKenna J, Peng T-L (2006). Revalençe od leisure-time physical activity in Taiwanese adultos: resultos of foru national serveys, 2000-2004. Prev Med 43(6): 454-457

21. Lee IM, Paffembarger RS, Hsiech C (1992). Physical activity and risk of prostatic câncer among college alumni. Am J Epidemiol 135(2): 169-179.

22. Luoto R, Latikka P, Pukkala E, Hakulienen T, Vihko V (2000). The effect of physical activity on breast cancer risk: a cohort study of 30,548 women. Eur J Epidemiol 16(10): 973-980.

23. Marcus BH (1995). Exercise behavior and estrategies for intervention. Res $Q$ Exerc Sport 66(4): 319-323.

24. Marcus BH, Nigg CR, Reibe D, Forsyth LAH (2000). Interactive communication strategies - implications for population-based physical-activity promotion. Am J Prev Med 19(2): 121-126.

25. Marcus BH, Williams DM, Dubbert PM, Sallis JF, King AC, Yancey AK, Franklin BA, Buchener D, Daniels SR, Claytor RP (2006). Physical Activity Intervention Studies: What We Know and What We Need to Know - A Scientific Statement From the American Heart Association Council on Nutrition, Physical Activity, and Metabolism (Subcommittee on Physical Activity); Council on Cardiovascular Disease in the Young; and the Interdisciplinary Working Group on Quality of Care and Outcomes Research. Circulation 114: 2739-2752.

26. Masson CR, Dias-da-Costa JS, Olinto MTA, Meneghel S, Costa CC, Bairros F, Hallal PC (2005). Prevalência de sedentarismo nas mulheres adultas da cidade de São Leopoldo, Rio Grande do Sul, Brasil. Cad Saúde Pública 21(6): 1685-1694.

27. Matsudo SM, Matsudo VKR, Araújo TL, Andrade DR, Oliveira L, Braggion G (2002). Nível de atividade física da população de São Paulo: análise de acordo com idade, nível sócio-econômico, distriguição geográfica e de conhecimento. Rev Bras Ciên Mov 10: 41-50.

28. McAuley D, McCrum E, Stott G, Evans A, Gamble R, McRoberts B (1998). Leves of physical activity, physical fitness and their relantionship in the northern ireland health and activity survey. Int J sports Med 19: 503-511.

29. Monteiro CA, Conde Wl, Matsudo SM, Matsudo VKR, Bonseñor IM, Lotufo PA (2003). A descriptive epidemiology of leisure-time physical activity in Brazil, 1996-1997. Rev Panam Salud Publica 14(4): 246-254.

30. Pate RR, Prat M. Blair SN, Haskell WL, Macera CA, Bouchard C, Buchner D, Ettinger W, Heath GW, King Ac (1995). Physical activity and public health : a recomendation from the Centers for Disease Control Prevention and Americam College of Sports Medicine. JAMA 273(2): 402-407.

31. Pitanga FJG, Lessa I (2005). Prevalência de fatores associados ao sedentarismo no lazer em adultos. Cad Saúde Pública 21(3): 870-877.

32. Prochaska JO, DiClemente CC, Noscross JC (1992). In search of how people change: applications to addictive behaviors. Am Psychologist 47(9): 1102-1114.

33. Sá GS, Duarte MFS (2005). Estágios de mudança de comportamento relacionados à atividade física em adolescentes. Rev Bras Med Esporte 11(2):104-108.

34. Salles-Costa R, Werneck GL, Lopes CS, Faerstein E (2003). Associação entre fatores sócio-demográficos e prática de atividade física de lazer no estudo pró-saúde. Cad Saúde Pública 
19(4):1095-1105.

35. Sallis JF (1994). Influences on physical activity of children, adolescents, and adults or determinants of active living. President's Council of Physical Fitness and Sports: Washington D.C. 2004 , series $1, \mathrm{n}^{\circ} 7$, august, 1-7.

36. Sallis JF, Hovell MF, Hosfstetter CR (1992), Predictors of adoption and maintenance of vigorous physical activity in men and women. Prev Med 21(2): 237-251.

37. Sallis JF, Owen N (1999). Physical Activity \& Behavioral Medicine. Califórnia: Sage Publications.

38. Sigal RJ, Kenny GP, Wasserman DH, Castaneda-Sceppa C, White RD (2006). Physical activity/exercise and type 2 diabetes. A consensus statemente form de Americam Diabetes Association. Diabete Care 29(6), 1433-1438

39. Spittaels H, De Bourdeaudhuij I, Vandelanotte C (2007). Evaluation of a website-devered computer-tailored intervention for increasing physical activity in the general population. Prev Med 44(3): 209-17.

40. Task Force on Community Preventive Services (2002). Recommendations to increase physical activity in communities. Am J Prev Med 22(4S): 67-72.

41. U.S. Department of Health and Human Services (1996). Physical activity and Health: a report of the Surgeon General. Atlanta, GA: U.S. Department of Health and Human Services. Centers for Disease Control and Prevention, National Center for Chronic Disease Prevention and Health Promotion, 85-259.

42. Word Health Organization (2003). WHO Global strategy on diet, physical activity and health: World Health Organization. http://www.who.int/dietphysical activity/pa/en/print.html $>[2007$ fev 02].

43. Yancey AK, Fielding JE, Flores GR, Sallis JF, McCarthy WJ, Breslow L (2007). Creating a Robust Public Health Infrastructure for Physical Activity Promotion. Am J Prev Med 32(1): 68-78. 